\title{
A PHD Filter for Tracking Multiple AUVs
}

\author{
José Melo, Aníbal Matos \\ INESC TEC (formerly INESC Porto) \\ Faculty of Engineering, University of Porto \\ Rua Roberto Frias, s/n, 4200-465 Porto, Portugal \\ \{jose.melo,anibal\}@fe.up.pt
}

\begin{abstract}
In this paper we address the problem of tracking multiple AUVs using acoustic signals. Using For this challenging scenario, we propose to use a Probability Hypothesis Density Filter and present a suitable implementation of the Sequential Monte Carlo PHD filter. It will be demonstrated that a particle filter implementation of the aforementioned filter can be used to successfully track multiple AUVs, changing in number over time, using range measurements from the vehicles to a set of acoustic beacons. Simulation results will be presented that allow to evaluate the performance of the filter.
\end{abstract}

\section{INTRODUCTION}

Autonomous Underwater Vehicles (AUVs) are becoming a reliable and cost-effective solution for performing tasks underwater in a fully automated way. Among the top tasks to be performed by these vehicles are bathymetric tasks, environmental surveying, surveillance or even mine countermeasures operations.

While most of these tasks are traditionally performed using not more than one vehicle simultaneously, there are significant research efforts focused towards the development of algorithms that allow fleets of AUVs to coordinate their efforts towards a common objective. With such developments, it is reasonable to expect that in a near future new applications will arise requiring the operation of multiple AUVs concurrently, cooperating to achieve a given goal.

The Localization and Navigation is one of the most fundamental problems for AUVs. The main navigation technology for current Autonomous Vehicles is dead reckoning, but deadreckoning alone is known to produce position estimates that will drift and grow unbounded over time. Bounding the errors of inertial navigation requires the use of some kind of navigational aids. For ground outdoor environments, GPS-based techniques can be used to solve the problem of localization, by providing position aids with high levels of precision. However for underwater environments, where the use of GPS is not an option, other sources of positional aids exist.

The two main sources of navigational aids for AUVs are Acoustic Navigation and Geophysical Navigation. Even though research on the latter, and specifically in Terrain Based Navigation techniques, has been producing promising results, Acoustic Navigation, on its various forms, still is the most widespread and reliable. For a more thorough review on recent AUV Navigation and Localization techniques, refer to [1].

Besides delivering position navigation aids, Acoustic Navigation methods also provide a way for an external acoustic receiver to track AUVs, by tapping the acoustic signals exchanged. In fact, this ability to externally track AUVs can be of crucial importance, particularly for application in the military and oil industry. In this article we are interested on using Acoustic Navigation, namely range-based One Way Travel Time (OWTT) techniques, for the external tracking of multiple AUVs. The main contribution of the work here presented is that we demonstrate how can a Sequential Monte Carlo implementation of a Probability Hypothesis Density (PHD) filters be effectively applied to the problem of tracking multiple AUVs, varying in number along the time.

PHD Filters are a fairly hot topic, in the sense that only recently have they been used to address multitarget tracking applications. Compared to more traditional approaches, like the Kalman Filter or even the Multiple Hypothesis Tracker, the main advantage of using set-based PHD Filters is the ability to skip the Data Association step, as it alleviates some of the computational burden involved. Additionally, it has also the potential to also reduce the complexity of the setup required for the acoustic navigation, which usually requires mutiplexing, whether in frequency or in time, the acoustic signals emitted by the different vehicles present in the acoustic network.

The remainder of this article is as follows. The different Acoustic Navigation alternatives are briefly covered on Section II, and in Section III the single target tracking models are introduced. Details on the FISST framework and derivation of the SMC PHD filter implementation can be found in Sections IV and V, respectively. Finally, on Section VI simulations results are presented that demonstrate the performance of the filter.

\section{Acoustic Navigation}

Acoustic Navigation embraces a number of techniques that rely on the exchange of acoustic signals between different acoustic sources, usually a vehicle and a series of acoustic beacons. From the time-of-flight of each of the acoustic signals it is possible for an acoustic receiver to compute whether ranges or ranges differences using, respectively, the times of arrival (TOA) or time differences of arrival (TDOA) of each signal. This process is obviously dependent on knowing the velocity of propagation of a sound wave in the water for a given location, which is usually a constant value around 1500 $\mathrm{m} / \mathrm{s}$. 
The localization problem using ranges only is usually referred to Spherical Localization, while Hyperbolic Localization refers to the localization problem using range differences. In this section a brief overview on the different possibilities will be provided, as this is relevant for the problem under discussion. Nevertheless, a detailed analysis on the problem of underwater navigation of robotic vehicles using acoustic positioning systems can be found in [2] and the references therein.

Acoustic Navigation systems are generally divided according to the size of the baseline, which is the distance between the beacons. Accordingly, Long Baseline (LBL), Short Baseline (SBL) and Ultra-Short Baseline (USBL) are the most common configurations used. While in SBL and USBL configurations the beacons are purely passive sensor, in the sense that they only listen to the acoustic signals emitted by the AUV, for LBL configurations the beacons are also required to emit. The communications between beacons and vehicle follow a predefined protocol, according to which each of the beacons sends an acoustic signal in response to the AUV. The AUVs is responsible to interrogate, in a predefined sequence, all the beacons that are part of the network. The different beacons are independently addressed using different acoustic signals, usually modulated whether in frequency or in time. In this way, the different participants of the acoustic network are easily distinguishable.

The main purpose for all of the different configurations mentioned is to know the position of an AUV relative to a set of acoustic beacons. Because in LBL systems the beacons also emit reply acoustic signals, in this configuration it is also possible to provide navigational aids to the vehicles. Recent advances in underwater communication topics have also brought Acoustic Modems to play a relevant part in underwater navigation capabilities. However, for shallow waters and adverse environmental conditions reliable underwater communications can be quite challenging, particularly for long distances.

LBL systems are one of the most for robust, reliable and accurate configurations of Acoustic Navigation systems, and are used in some of the more challenging scenarios, like in the military and oil industry. The reason for this is because LBL systems allow to simultaneously externally track one vehicle and provide positioning aids to it. For those reasons, on the remainder of this article particular attention will be given to these systems. In specific the focus will be on using GPS Intelligent Buoys (GIBs) as acoustic beacons, implementing a scheme that is also known as Inverted LBL. Compared to traditional LBL systems, using GIBs as beacons is more convenient because the beacons don't need to be deployed to the sea bottom, which can be particularly cumbersome.

\section{A. Navigation and Tracking of Multiple AUVs}

LBL algorithms provide navigational aids to AUVs in terms of ranges to each one of the beacons that compose the network. Standard implementations depend on the AUV independently sending a query acoustic signal to each one of the beacons and waiting for their reply. The time difference between sending the query and receiving the reply, also known as Two Way Travel Time (TWTT), is then converted to range between AUV and beacon. After this step, any given Spherical Localization algorithm can be implemented in a trivial way.

A simple solution has been proposed for scaling LBL algorithms to provide navigational aids to multiple vehicles, which is named One-Way-Travel-Time (OWTT) Acoustic Navigation. OWTT techniques require a low-drift clock synchronization between all the vehicles and beacons present in the acoustic network. For this arrangement, beacons are configured to send periodic acoustic signals at specific time instants, while the vehicles are purely passive. OWTT rely on a protocol which defines the exact instant on which each of the vehicles is supposed to emit its acoustic signal. Because all the clocks are synchronized, the time elapsed from the time instant the beacon is supposed to transmit until each of the vehicles detects it (the One Way Travel Time), can be directly used to compute ranges. In this configuration the different beacons are required to transmit different signals, which is commonly implemented as signals with different frequencies. Alternatively, and because the number of the beacons is small enough (usually lower than 4), Time Division Multiple Access (TDMA) techniques can also be used. For further details on OWTT refer to [3].

Tracking a single vehicle when using standard LBL systems can be done by eavesdropping the acoustic signals in the network, as described in [4]. However, scaling up these system for multiple vehicles has no easy and standard solution. Tracking a vehicles requires it not to be passive, meaning that the vehicle needs to send acoustic signals. At the same time traditional approaches require that the buoys have an explicit way to associate the signals they can listen to specific vehicles. For those purposes, different frequency modulations are usually employed.

Time modulation approaches, similar to TDMA techniques, can be used but the drawback of this is that it introduces significant delays and, therefore degrades the performance of the navigation aid and tracking accuracies. The other alternative would be using different signals (e.g. distinct frequencies) for each of the vehicles. However this is likely not an interesting solution mainly due to the necessary increment in complexity of the hardware needed as the number of vehicles to track increases. Furthermore, each vehicle would need to have specific hardware configurations which can be very costly.

Up to the authors knowledge, there aren't any standard solutions for simultaneous navigational aid and external tracking of multiple vehicles. The reason for this is that, as described, existing Acoustic Navigations techniques like LBL don't scale up well as the number of vehicles increases. With no other obvious options to be able to physically associate each acoustic signal with a given vehicle, in this article we try do address the problem in a different way, by using an appropriate multitarget estimation framework able to cope with such peculiarities.

In specific, we propose to address the problem of acoustically tracking multiple AUVs while at the same time maintain- 
ing the possibility to provide navigational aids. The conceptual scenario is to have the acoustic beacons to synchronously send an acoustic ping using in a similar way to One Way Travel Time (OWTT) techniques. With the vehicles knowing the mapping between buoys and transmitting frequencies, computing their own position is trivial. On the other hand, and similarly to LBL, all the vehicles would also synchronously send an acoustic signal to be interpreted by the buoys, but the novelty here is that all the vehicles would use the same acoustic signal. Because PHD Filters don't require the data association step between a given measurements and targets, usually required on other multitarget tracking algorithms, they seem to be adequate to the problem in discussion. In this way, it is possible to develop an Acoustic Navigation system compatible with simultaneous navigational aid and target tracking of multiple vehicles. The details on such filter and their implementation are going to be made clear in the following sections.

\section{TRACKING A SINGLE TARGET}

Externally tracking an AUV by tapping the acoustic signals exchanged between the vehicle and a set of acoustic buoys in an LBL configuration is a well known and previously studied problem, which has already been addressed.

For the sake of clarity, in this section we will briefly describe the motion and sensor models used for the single target tracking problem, as well as provide a description of the setup involved. Nevertheless, the concepts here presented also hold for the case of tracking multiple underwater vehicles. The interested reader should refer to [4] and the references therein for a comprehensive coverage on this topic.

The scenario here in analysis can be described as the case of tracking a single underwater target in using a set of buoys in an LBL configuration. The setup involved consists not only on the vehicle, an Autonomous Underwater Vehicle, but also on a set of three acoustic beacons, or buoys. It is considered that the length of the baselines, which is the distance between each of the buoys, is long enough so that the depths that the AUVs navigates are considered to be negligible and, thus, we only consider the two-dimensional motion of the AUV on the horizontal plane. If this wouldn't be the case, then an additional acoustic buoy would be required, but the underlying principles would still apply. Figure 1 presents a schematic of the setup required for tracking external AUVs in a scenario as the one described.

It is also considered the clocks sources of both the vehicles and the set of buoys are synchronous and with drifts small enough so that OWTT techniques can be used through the entire duration of the missions. What this assumption means is that all the systems, buoys and vehicle, share a common clock source and are aware of the exact time instant each of the systems emits a given acoustic signal. While this scenario is compatible with the navigational aids being provided to the vehicle, in the work here being presented we are only concerned with the external tracking of the vehicle. Having that specific application in mind, we are only considering the

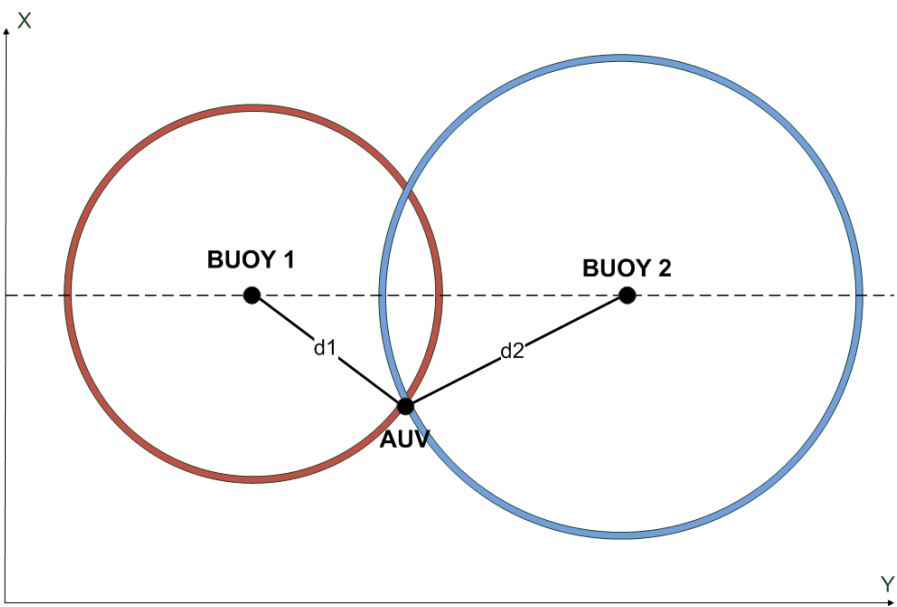

Fig. 1. Schematic view of the setup required for tracking external AUVs

acoustic signals emitted by the AUVs, synchronously and at a frequency of $1 \mathrm{~Hz}$. Even it is desirable that observations of the target to happen frequently, this value is common on systems employing OWTT techniques mostly for practical reasons: it is relatively easy to take advantage of the GPS Pulse Per Second (PPS) for clock synchronization schemes.

With this setup, it is possible to directly compute ranges to each of the beacons from the time-of-flight that each of the beacon detects. Because the clock sources are synchronous, and the time instants on which the vehicle is supposed to emit its acoustic signals are fixed and known to the entire system, the time-of-flight will be equivalent to the delay between the emission time instant, and the time instant when each of the buoys detects the received signal.

\section{A. Target and Sensor Model}

The system that was just described in the previous section consists on an AUV navigating and range observations of this vehicle are obtained by the three acoustic beacons, deployed on specific positions that are known. This scenario configures a typical target tracking applications. The range observations are naturally noisy and don't any kind of data association with targets, which means that then can not distinguish from which target each of the measurements was originated. Additionally, the possibility of a missed detections is also considered. The behaviour of the whole system can be described by the means of the single target dynamical model, $f_{k}$ and the single target measurement model, Equations (1) and (2), respectively.

$$
\begin{gathered}
\mathbf{x}_{k}=f_{k}\left(\mathbf{x}_{k-1}, \mathbf{v}_{k-1}\right) \\
\mathbf{z}_{k}=h_{k}\left(\mathbf{x}_{k}, \mathbf{n}_{k}\right)
\end{gathered}
$$

In the equations above, $\mathbf{x}_{k}$ refers to the state vector of a target. For the remainder of the work here presented, the state vector $\mathbf{x}_{k}$ is defined as

$$
\mathbf{x}_{k}=\left[\begin{array}{lll}
x_{k} & y_{k} & \psi_{k}
\end{array}\right]^{T}
$$


where $\left[x_{k}, y_{k}\right]^{T}$ denotes the position and $\psi_{k}$ the target's heading. It is further assumed that the dynamic model for all the targets follows a Gaussian constant surge velocity model, according to (4).

$$
\mathbf{x}_{k}=\mathbf{x}_{k-1}+\left\{\begin{array}{c}
|v| \Delta \cos (\psi) \\
|v| \Delta \sin (\psi) \\
0
\end{array}\right\}+\mathbf{v}_{k-1}
$$

In the equation above $\Delta$ is the sampling interval, $|v|$ is the surge velocity of the vehicle, which is assumed to be constant and equal for every target, and $\mathbf{v}_{k-1} \sim \mathcal{N}(0, Q)$ is the white Gaussian process noise, with $Q$ as the process noise covariance.

On a given sampling period interval, each of the acoustic beacons produces one range observation of the target, that is related with the state vector $\mathbf{x}_{k}$ according to the measurement equation in

$$
z_{k, i}=h_{i}\left(\mathbf{x}_{k}\right)+\mathbf{n}_{k, i}
$$

where $h_{i}$ is the real valued function responsible for taking the range measured by each acoustic beacon, $r_{i}$, with a known fixed position $\left(x_{0, i}, y_{0, i}\right)$ and compared to the expected range corresponding to the estimated position of the vehicle. $\mathbf{n}_{k, i} \sim$ $\mathcal{N}\left(0, \sigma_{i}\right)$ is the measurement noise in acoustic beacon $i$.

$$
h_{i}\left(\mathbf{x}_{k}\right)=r_{k, i}-\sqrt{\left(x_{k}-x_{0, i}\right)^{2}+\left(y_{k}-y_{0, i}\right)^{2}}
$$

\section{RFS AND THE PHD FILTER}

The Finite Set Statistics (FISST) framework, developed mainly by Mahler [5], builds up on random set theory, and provides a unified mathematical framework that supports, among other applications the general Multisource-Multitarget Bayes Filter (7-8). This filter is a set valued version of the Bayes Filter.

$$
p\left(\mathbf{X}_{k} \mid \mathbf{Z}^{k}\right)=\frac{p\left(\mathbf{Z}^{k} \mid \mathbf{X}_{k}\right) p\left(\mathbf{X}_{k} \mid \mathbf{Z}^{k-1}\right)}{p\left(\mathbf{Z}^{k} \mid \mathbf{Z}^{k-1}\right)}
$$

where

$$
p\left(\mathbf{Z}^{k} \mid \mathbf{Z}^{k-1}\right)=\int p\left(\mathbf{Z}_{k} \mid \mathbf{X}\right) p\left(\mathbf{X} \mid Z^{k}\right) \delta \mathbf{X}
$$

Analogously to the single target traditional recursive Bayes Filter, the multisensor-multititarget Bayes filter propagates a multitarget Bayes posterior distribution through time. For the latter case, the multitarget state (9) and the multitarget measurements (10) are modelled as Random Finite Sets (RFS).

$$
\begin{gathered}
\mathbf{X}_{k}=\left(\bigcup_{x \in \mathbf{X}_{k-1}} \mathbf{S}_{k \mid k-1}(\mathbf{x})\right) \cup\left(\bigcup_{\mathbf{x} \in \mathbf{X}_{k-1}} \mathbf{B}_{k \mid k-1}(\mathbf{x})\right) \cup \Gamma_{k} \\
\mathbf{Z}_{k}=\mathbf{K}_{k} \cup\left(\bigcup_{z \in \mathbf{Z}_{k}-1} \Theta_{k}(\mathbf{x})\right)
\end{gathered}
$$

Because the usage of random sets, as opposed to random vectors, situations like target a varying number of targets or even (dis)appearance and spawning, clutter measurements, extended targets, false alarms and missed detections can be easily addressed. Note that in (7) $\mathbf{S}_{k \mid k-1}$ is a model for the targets that survive from the previous time step, $\mathbf{B}_{k \mid k-1}$ is a model for spawned targets, and $\Gamma_{k}$ is a model for new targets appearing in the new time step. Similarly, in (10) $\mathbf{K}_{k}$ is a model for the clutter intensity among the measurements, and $\Theta_{k}$ is the model for measurements observed due to the present targets, including the probability of a missed detections.

The data association step required for traditional multitarget tracking algorithms, like the multiple hypothesis tracking (MHT) or the joint probabilistic data association (JPDA), can be computationally expensive, particularly when the number of targets is large. Because FISST based filters have no data association, they present an obvious advantage over their counterparts.

The first moment of an RFS, $\mathbf{D}_{k}$, is known as intensity function or Probability Hypothesis Density. The PHD Filter, initially proposed in [6] propagates only the first-order statistical moment of the RFS of states, instead of propagating the full multi-target posterior. In a way, the PHD filter is the set valued counterpart of constant gain Kalman filter for the random vector case. Such approximation makes the multitarget Bayes filter computationally tractable. However, for this approximations to hold, some assumptions must be observer, namely the signal to noise ratio (SNR) has to be high and all the targets should move independently of each other.

A probability hypothesis density function is completely characterized by the property in (11), which means that integrating a given PHD function $\mathbf{D}_{k}$ over the entire set of state spaces gives the estimated number of elements within the set. Additionally, the peaks of $\mathbf{D}_{k}$ identify the likely position of the targets.

$$
\int_{S} \mathbf{D}_{k \mid k}\left(\mathbf{X} \mid \mathbf{Z}^{(k)}\right) d \mathbf{X}
$$

The PHD filter predictor equations are given by (12-13) while the PHD filter corrector equations are in (14-15). These equations are the core of the general PHD filter. From the different possibilities to implement these equations, we chose to use the Sequential Monte Carlo (SMC) PHD, particularly because of the non-linear sensor and target models. The details of such implementation will be provided in the following section.

$$
\begin{aligned}
\mathbf{D}_{k \mid k-1}\left(\mathbf{X} \mid \mathbf{Z}^{k}\right)= & \gamma_{k}\left(\mathbf{x}_{k}\right)+ \\
& \int \phi_{k \mid k-1}\left(\mathbf{x}_{k-1}\right) \mathbf{D}_{k \mid k}\left(\mathbf{X} \mid \mathbf{Z}^{k}\right) d_{\mathbf{x}_{k-1}} \\
\phi_{k \mid k-1}\left(\mathbf{x}_{k-1}\right)= & p_{S, k}\left(\mathbf{x}_{k-1}\right) f_{k \mid k-1}\left(\mathbf{x}_{k} \mid \mathbf{x}_{k-1}\right)
\end{aligned}
$$




$$
\begin{gathered}
\mathbf{D}_{k \mid k} \cong \mathbf{L}_{\mathbf{Z}_{k+1}}(\mathbf{X}) \mathbf{D}_{k}\left(\mathbf{X} \mid \mathbf{Z}^{k}\right) \\
\mathbf{Z}_{k+1}=\left(1-p_{D}(\mathbf{X})\right)+ \\
\sum_{Z \in \mathbf{Z}_{k}} \frac{p_{D}(\mathbf{X}) L_{Z}(\mathbf{X})}{\lambda_{k} c_{k}(Z)+\int p_{D} L_{Z}(\mathbf{X}) \mathbf{D}_{k \mid k-1} d \mathbf{X}}
\end{gathered}
$$

In the equations above, $p_{S, k}$ refers to the probability a given target has to survive, to one time step to the immediately after, and $f_{k \mid k-1}$ refers to the target transition density. Moreover, $\gamma_{k}$ refers to the intensity of spontaneous births, $c_{k}$ to the clutter spatial distribution and $\lambda_{k}$ to the clutter rate. Also, $p_{D}$ is the probability of detection of a target, and $L_{Z}$ the measurement likelihood.

\section{The Sequential Monte Carlo PHD (SMC-PHD) FILTER}

A closed formed solution for the PHD filter (12-15), has been derived in by Vo [7]. This filter, the Gaussian Mixture PHD filter (GM-PHD) admits only scenarios on which the targets evolve and generates observations independently, and following a linear and Gausian dynamical model and, additionally, that the sensors as well follow linear and Gaussian measurement models. Recalling the single target scenario described in Section III, there are non-linearities present both in the target dynamical model (1) and measurement model (2), and thus this is not a suitable approach. Because of that, an approximation to the PHD filter recursion is more adequate.

The Sequential Monte Carlo PHD filter is an approximation to general PHD recursion that, analogously to standard Particle Filters, uses random distributed particles to approximate the density functions that represent PHD predictor and corrector equations. For that reason, another possible designation for the SMC-PHD is Particle PHD filter. In fact, for the case when there is only one target with no birth, no death, no clutter and unity probability of detection, the PHD filter reduces to the standard particle filter.

Considering the particle approximation,the PHD predictor equation can be rewritten as in (16), where the approximations is done with $L_{k-1}$ particles, corresponding to the RFS containing the surviving targets, and $J_{k}$ new particles introduced, representing the RFS of the birth targets. For the general case the birth particles should cover the entire space of observation however, it is often the case that the prior knowledge regarding the location where possible new targets may appear is incorporated.

$$
\mathbf{D}_{k \mid k-1}\left(\mathbf{x}_{k}\right)=\sum_{i=1}^{L_{k-1}+J_{k}} w_{k \mid k-1} \delta_{\mathbf{x}_{k-1}^{(i)}}(\mathbf{x})
$$

where

$$
\mathbf{x}_{k \mid k-1} \sim \begin{cases}q_{k}\left(\cdot \mid \mathbf{x}_{k-1}, Z_{k}\right), & \text { if } 1 \leq i \leq L_{k-1} \\ p_{k}\left(\cdot \mid Z_{k}\right), & \text { if } L_{k-1} \leq i \leq J_{k}\end{cases}
$$

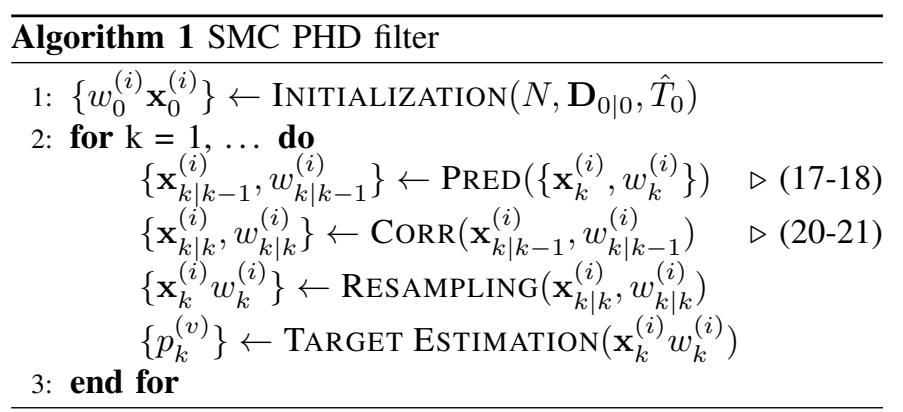

and

$$
w_{k \mid k-1}= \begin{cases}\frac{\phi_{k \mid k-1}\left(\mathbf{x}_{k}^{(i)} \mid \mathbf{x}_{k}^{(i)}-1\right)}{q_{k}\left(\mathbf{x}_{k}^{(i)} \mid \mathbf{x}_{k-1}^{(i)}, Z_{k}\right)} w_{k-1}, & \text { if } 1 \leq i \leq L_{k-1} \\ \frac{\gamma_{k}\left(\mathbf{x}_{k}^{(i)}\right)}{J_{k} p_{k}\left(\mathbf{x}_{k}^{(i)} \mid Z_{k}\right)}, & \text { if } L_{k-1} \leq i \leq J_{k}\end{cases}
$$

In the same way, the SMC approximation for the PHD corrector can be rewritten as in (19)

$$
\mathbf{D}_{k \mid k}\left(\mathbf{x}_{k}\right)=\sum_{i=1}^{L_{k-1}+J_{k}} w_{k \mid k} \delta_{\mathbf{x}_{k-1}^{(i)}}(\mathbf{x})
$$

where

$w_{k \mid k}=\left[\left(1-p_{D}\left(\mathbf{x}_{k}^{(i)}\right)+\sum_{z \in Z_{k}} \frac{p_{D}\left(\mathbf{x}_{k}^{(i)}\right) g_{k}\left(z \mid \mathbf{x}_{k}\right)}{\mathbf{K}_{k}+c_{k}(z)}\right] w_{k \mid k-1}\right.$

$\mathbf{K}_{k}$ being the intensity of the clutter measurement RFS and

$$
c_{k}=\sum_{i=1}^{L_{k-1}+J_{k}} p_{D}\left(\mathbf{x}_{k}^{(i)}\right) g_{k}\left(z \mid \mathbf{x}_{k}\right) w_{k \mid k-1}
$$

The particle transition density $\phi_{k \mid k-1}$ and measurement likelihood $g_{k}\left(z \mid \mathbf{x}_{k}\right)$ are obtained reusing the previously derived single target dynamical model (4) and measurement model (5), respectively. Further details on the derivation and convergence properties of the SMC-PHD filter can be found in [7], [8]

The pseudo-code for the SMC-PHD recursion is provided in Algorithm 1. The recursive algorithm can be can be informally described by the three different stages: prediction, correction and resampling. On the prediction stage, each of the $L_{k-1}$ particles that survived from the previous time step is propagated, and an additionally, $J_{k}$ birth particles are introduced. All the $L_{k-1}+J_{k}$ are weighted according to (18). Following the prediction stage, in the corrector stage all the particles are weighted according to the measurement set (20).

Because in the prediction stage of the algorithm there are always a number $L_{k-1}$ of birth particles that are introduced, the number of particles is always increased on every time step of the filter. In the resampling stage of the algorithm the number of particles in the filter is downscaled to a number that is proportionally to the total particle mass $\hat{N}_{k \mid k}$. 


\begin{tabular}{|c|c|}
\hline Parameter & Value \\
\hline Filter Settings & \\
\hline $\begin{array}{l}\text { Particles Per Target }(\mathrm{N}) \\
\text { Particles Per Birth }(\mathrm{M}) \\
\text { Predictor Settings }\end{array}$ & $\begin{array}{c}1000 \\
500\end{array}$ \\
\hline $\begin{array}{l}\text { Prob. of Survival }\left(p_{s}\right) \\
\text { Prob. of Birth }\left(p_{b}\right) \\
\text { Process Noise Variance }\left(\sigma_{x, y}^{2}\right) \\
\text { Process Noise Variance }\left(\sigma_{\psi}^{2}\right) \\
\text { Corrector Settings }\end{array}$ & $\begin{array}{c}0.95 \\
0.005 \\
1.2 \\
10^{-2}\end{array}$ \\
\hline $\begin{array}{l}\text { Prob. of Detection }\left(p_{d}\right) \\
\text { Measurement Noise Variance }\left(\sigma_{r, B}^{2}\right) \\
\text { Clutter Intensity }\left(\lambda_{k}\right)\end{array}$ & $\begin{array}{c}0.98 \\
1 \\
\left(a^{3}-1\right) / V \\
a=\operatorname{floor}\left(\left|\mathbf{Z}_{k}\right|\right)\end{array}$ \\
\hline
\end{tabular}

SMC-PHD PARAMETERS USED FOR THE SIMULATIONS

$$
\hat{N}_{k \mid k}=\sum_{i=1}^{L_{k-1}+J_{k}} w_{k \mid k}^{(i)}
$$

The filter estimate for the total number of targets is given by the nearest integer of the to the total particle mass, $\operatorname{int}\left(\hat{N}_{k \mid k}\right)$. The resampling stage also prevents situations of sample impoverishment. The usual resampling strategies for particles filters can be used, see [9] for an overview. The resampling stage of the SMC-PHD filter differs only from the traditional resampling strategies adopted in that in the PHD filter the weights are not normalized to sum up to one but, instead,to the expected number of targets [8].

Though not a integral part of the original SMC-PHD algorithm, target estimation plays an important role as it is the stage where the locations of each of the targets are obtained. A way of extracting the targets, is to estimate the number of present targets in the current time-step, and then perform k-means clustering. Another alternative would be to fitting a Gaussian Mixture Model to the particles of the current time-step. While in principle any general clustering techniques could, there has been a strong preference of the community on using the two methods mentioned.

\section{Simulation Results}

In this section, we present simulation results of the implemented SMC-PHD filter for tracking multiple Autonomous Underwater Vehicles using range measurements. In specific, simulations considering scenarios three vehicles will be presented. Each of the vehicles under consideration follow a linear Gaussian dynamics as the one described in (4). On the other, a set of three acoustic buoys, referred to $B_{1}, B_{2}$, and $B_{3}$, throughout the remainder of this obtain the range measurements, according to its own predefined positions. The ranges are corrupted with Gaussian noise. An image of the simulated trajectories can be seen in Figure 2. Targets $t_{1}$ appears at instant $t_{i, 1}=10 \mathrm{~s}$, an disappears at time instant $t_{f, 1}=240 \mathrm{~s} 10$; conversely, target $t_{2}$ appears at $t_{i, 2}=75$ and disappears at instant $t_{f, 2}=230$; finally target $t_{3}$ appears at instant $t_{i, 1}=145 \mathrm{~s}$, an disappears at time instant $t_{f, 1}=220 \mathrm{~s}$.

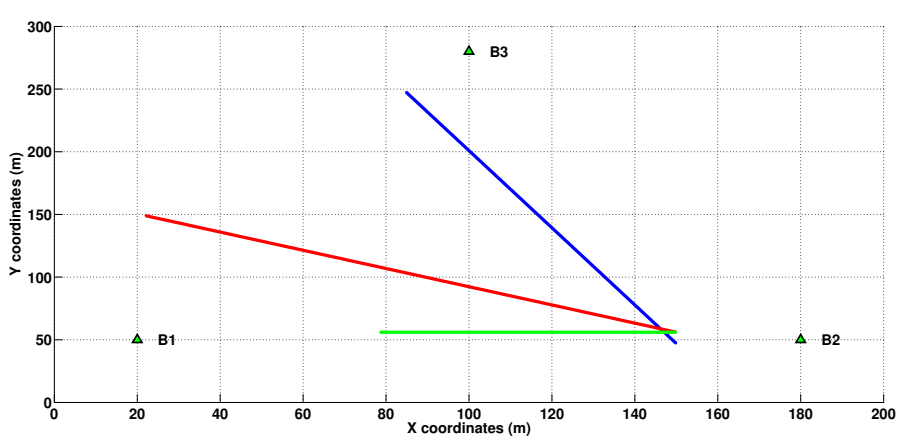

Fig. 2. Trajectories of targets $t_{1}, t_{2}$, and $t_{3}$, in blue, red and green, respectively

The general SMC-PHD filter assumes that new targets can birthed throughout the entire observation space. Though convenient assumption, this assumption means having an enough number of newborn particles drawn from a uniform density across the whole state-space. In this implementation an alternative path was chosen, as it is reasonable to assume that for applications where multiple AUVs are used, the positions from where the vehicles are usually launched in the water are known. For this reason it was assumed that in the simulations in analysis new targets can appear spontaneously according to a Poisson Point Process with intensity function $\gamma=\mathcal{N}\left(. ; x_{\gamma}, Q_{\gamma}\right)$ where $x$ and $Q$ represent the center and variance of the location where AUVs are launched.

$$
x_{\gamma}=[150,50, \pi]^{T} \text { and } Q_{\gamma}=\operatorname{diag}\left(\sigma_{\gamma, x}^{2}, \sigma_{\gamma, y}^{2}, \sigma_{\gamma, \psi}^{2}\right)
$$

The average of number clutter measurements, the RFS $\mathbf{K}_{k}$, is assumed to be uniform over the surveillance region and, thus, modelled as

$$
\mathbf{K}_{k}=\lambda_{c, k} V u(z)
$$

where $\lambda_{c, k}$ is the average number of clutter returns per unit region, $V$ is the surveillance region and $u(z)$ is the uniform density over the surveillance region. For every acoustic signal each underwater vehicle sends, there will be a number of measurements equal to the number of detections in beacons. In this case there can be three, if all the beacons are able to detect the signal, or less range measurements. Because there is to association between detections and targets, the detections from all the beacons have to be combined, to accommodate and adequate observation state. Because there are three beacons, on average the number of clutter measurements is $(\# A U V)^{3}-1$, which is cubical in the number of targets to track.

Figure 3 illustrates the tracking performance of the filter for three targets. As it can be seen, despite some false alarms on the presence of non-existent targets the filters is able to 

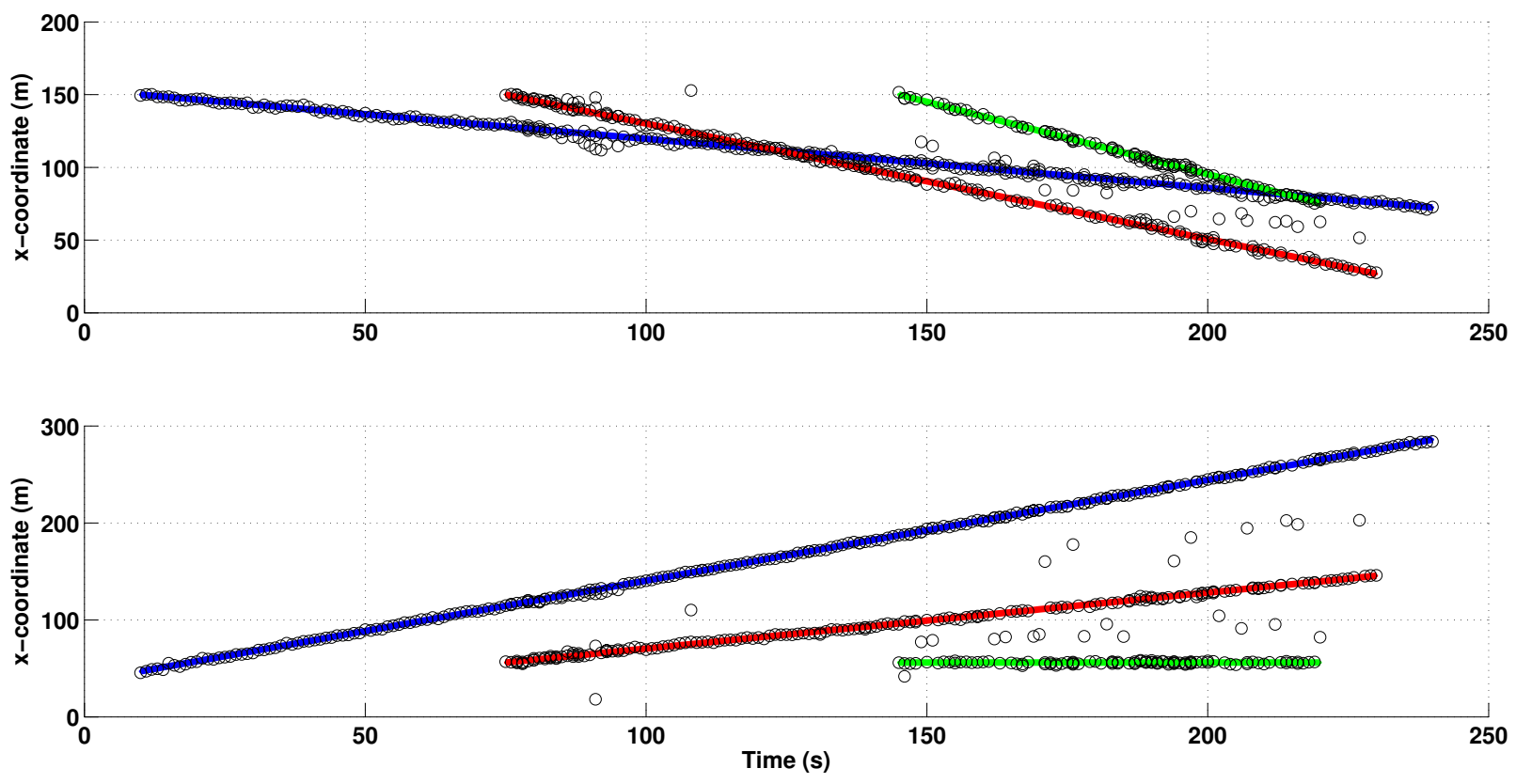

Fig. 3. Position Estimates for the PHD filter

correctly track all the targets through the entire duration of the simulation. Figure 4 demonstrates the results of the SMC-PHD filter in terms of the estimated cardinality of the filter, that is, the estimated number of vehicles. According to the figure, there are some variations in estimated number of targets. There variations are particularly visible whenever new targets are detected. Comparing the accuracy of the position estimates, which somehow contrasts with the variations in the cardinality of the filter, it is possible to infer sometimes the ghost targets are overlapped with targets that actually exist.

Up to this point not much have been said about both the Resampling and Target Estimation stages of the derived SMC-PHD filter. In fact, not much focus has been put on this two stages, and widely known algorithms have been used. For the Resampling strategy, a Systematic Resampling Algorithm was used, they have been to shown to outperform others in therms of resampling quality and computational complexity [9]. As for the the Target Estimation stage, a

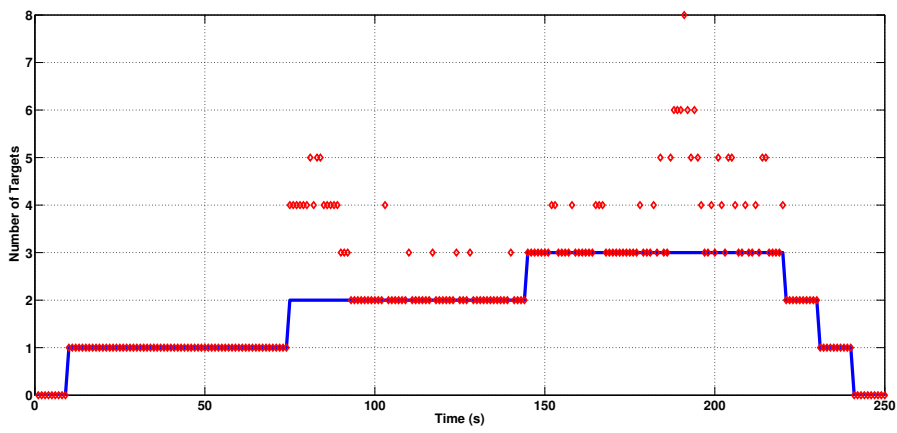

Fig. 4. Trajectories of target $t_{1}, t_{2}$, and $t_{3}$, in blue, red and green, respectively standard k-Means algorithm was preferred, with a maximum of 50 iterations to convergence. Table I lists the parameters used for the implemented SMC-PHD filter, and they values they take during simulation. The filter was implemented in Maltab software and performs well under the simulation time. Nevertheless if the number of targets increases significantly the computational complexity is likely to be severely affected.

\section{CONCLUSION}

The work here presented constitutes a preliminary study on the use of set-based PHD filters for tracking multiple AUVs. Operations with multiple vehicles are likely to become very appealing in a near future, not only in terms of flexibility and efficiency, but also in terms of performing a set task that otherwise wouldn't be possible. While the problem of providing navigational aids to multiple vehicles has been addressed numerous times, tracking multiple AUVs is a question that has been overlooked and is still open.

In this article it was demonstrated, that the problem of tracking of multiple AUVs can be tackled using PHD filters. Up to the authors knowledge, this is the first time that a similar approach has been proposed. Because THE PHD filter doesn't require a specific association between measurements and the targets that produced them, this approach can be very interesting for tracking multiple underwater vehicles. By using a PHD filter, there is no need on developing intricate strategies to distinguish vehicles like complex hardware of modulation strategies.

While the tracking performance of the filter has been very encouraging, there are some issues that still need to be address, namely the variation on the cardinality estimates. Future directions will include the study of suitable alternatives to tackle such issue. While Cardinalised-PHD filters have been 
referred in the literature to address this problem, they also come with a cost in terms on computational complexity. On the other, a more robust algorithm can probably be attained by deriving appropriate gating strategies for the measurements or even track continuity/management schemes.

\section{ACKNOWLEDGMENT}

This work is financed by the ERDF - European Regional Development Fund through the COMPETE Programme (operational programme for competitiveness) and by National Funds through the FCT - Fundação para a Ciência e a Tecnologia (Portuguese Foundation for Science and Technology) within project «FCOMP-01-0124-FEDER-037281».

The first author was supported by the Portuguese Foundation for Science and Technology through the Ph.D. grant SFRH/BD/70727/2010.

\section{REFERENCES}

[1] L. Paull, S. Saeedi, M. Seto, and H. Li, "Auv navigation and localization A review," Oceanic Engineering, IEEE Journal of, vol. 39, no. 1, pp. 131-149, Jan 2014.
[2] A. A. Peñas, "Positioning and navigation systems for robotic underwater vehicles," Ph.D. dissertation, UNIVERSIDADE TECNICA DE LISBOA, INSTITUTO SUPERIOR TECNICO, 2009.

[3] R. Eustice, L. Whitcomb, H. Singh, and M. Grund, "Experimental results in synchronous-clock one-way-travel-time acoustic navigation for autonomous underwater vehicles," in Robotics and Automation, 2007 IEEE International Conference on, April 2007, pp. 4257-4264.

[4] J. Melo and A. Matos, "Guidance and control of an asv in auv tracking operations," in OCEANS 2008, Sept 2008, pp. 1-7.

[5] R. Mahler, "Random set theory for multisource-multitarget information fusion," in Handbook of Multisensor Data Fusion, M. E. Liggins, D. L. Hall, and J. Llinas, Eds. Taylor \& Francis Group, 2008.

[6] _ - "Multitarget bayes filtering via first-order multitarget moments," Aerospace and Electronic Systems, IEEE Transactions on, vol. 39, no. 4, pp. 1152-1178, Oct 2003.

[7] B.-N. Vo, S. Singh, and A. Doucet, "Sequential monte carlo methods for multitarget filtering with random finite sets," Aerospace and Electronic Systems, IEEE Transactions on, vol. 41, no. 4, pp. 1224-1245, Oct 2005.

[8] D. E. Clark, "Multiple target tracking with the probability hypothesis density filter," Ph.D. dissertation, HERIOT-WATT UNIVERSITY, 2006.

[9] J. Hol, T. Schon, and F. Gustafsson, "On resampling algorithms for particle filters," in Nonlinear Statistical Signal Processing Workshop, 2006 IEEE, Sept 2006, pp. 79-82. 